\title{
QUASICLASSICAL THEORY \\ OF SPIN-VALVE MAGNETORESISTANCE: ROLE OF SPIN-FLIP SCATTERING
}

\begin{abstract}
O.M. BAKSALARY* AND J. BARNAŚ
Magnetism Theory Division, Institute of Physics, A. Mickiewicz University Matejki 48/49, 60-769 Poznań, Poland

The Boltzmann kinetic equation is used to analyse the in-plane electronic transport in magnetic multilayers. Both diffuse and electron-momentum-conserving spin-flip scattering processes are included. Numerical results show that the momentum-conserving scattering processes reduce the spin-valve magnetoresistance.
\end{abstract}

PACS numbers: $72.15 . \mathrm{Gd}, 73.40 . \mathrm{Jn}$

Theoretical interpretations of the spin-valve magnetoresistance (SVMR) in magnetic multilayers are usually based on the two-current model [1-3]. In the simplest version of the model one assumes a parabolic electron band for the conduction electrons ( $s$-band) and a narrow band for the magnetic electrons ( $d$-band) and one neglects a contribution of the latter band to the electrical conductivity. At low temperatures the two spin channels for electronic transport can be considered as independent, but at higher temperatures spin-mixing scattering processes have to be taken into account [4-6]. In this paper we use the Boltzmann kinetic equation to describe SVMR in magnetic sandwich structures for an electric current flowing in the film plane. Both diffuse and electron-momentum-conserving (coherent) spin-flip scattering processes are taken into account.

Consider a symmetrical structure, in which two ferromagnetic films of thickness $d_{\mathrm{m}}$ are separated by a nonmagnetic spacer of thickness $d_{\mathrm{n}}$. Let the electronic potential $V_{\mathrm{m}}$ for the conduction electrons in the magnetic films be independent of the spin orientation, but generally different from the electronic potential $V_{\mathrm{n}}$ in the spacer.

If the axis $z$ is normal to the film plane, then the Boltzmann kinetic equation for the $i$-th layer $(i=1,2,3)$ can be written in the form [4]

$$
\frac{\partial g_{i \sigma}(z, v)}{\partial z}+\frac{g_{i \sigma}(z, v)}{v_{z} \tau_{i \sigma}}+\frac{g_{i \sigma}(z, v)-g_{i-\sigma}(z, v)}{v_{z} \tau_{i+-}}=\frac{|e| E}{m v_{z}} \frac{\partial f_{i}^{0}(v)}{\partial v_{x}}
$$

where $g_{i \sigma}(z, v)$ is the deviation of the Fermi-Dirac distribution function in the $i$-th layer from the equilibrium distribution $f_{i}^{0}(v)$. In the above equation $\tau_{i \sigma}$ is the spin-dependent relaxation time, which in the ferromagnetic films $(i=1,3)$ is

\footnotetext{
*Corresponding author.
} 
equal to $\tau_{\mathrm{m}+}$ and $\tau_{\mathrm{m}-}$ respectively for the spin antiparallel and parallel to the local magnetization axis, whereas in the nonmagnetic spacer $(i=2)$ it is independent of the spin orientation, $\tau_{2 \sigma}=\tau_{\mathrm{n}}$. Finally, $\tau_{i+-}$ is a spin-mixing relaxation time, which is equal to $\tau_{\mathrm{m}+-}$ in the magnetic films and $\tau_{\mathrm{n}+-}$ in the nonmagnetic spacer. Diffuse spin-flip scattering processes contribute to $\tau_{i \sigma}$, whereas the coherent ones to $\tau_{i+-}$.

A general analytical solution of Eq. (1) can be found in Ref. [6]. The solutions in individual layers have to obey some boundary conditions at all interfaces and surfaces. Consider first the interfaces between the magnetic films and the spacer. If $T_{i j}^{0}$ is the probability that an electron with energy $\mathcal{E}$, incident from the layer $i$ on a perfectly flat interface, will be transmitted to the adjacent layer $j$ and $R_{i j}^{0}$ is the probability that it will be reflected from the layer $j$ back to the layer $i$, then $R_{i j}^{0}$ and $T_{i j}^{0}$ are given by the formulae [7]:

$$
\begin{aligned}
& R_{i j}^{0}\left(\mathcal{E}, u_{i}\right)=\left|\frac{1-h_{i j}\left(\mathcal{E}, u_{i}\right)}{1+h_{i j}\left(\mathcal{E}, u_{i}\right)}\right|^{2}, \\
& T_{i j}^{0}\left(\mathcal{E}, u_{i}\right)=1-R_{i j}^{0}\left(\mathcal{E}, u_{i}\right),
\end{aligned}
$$

where

$$
h_{i j}\left(\mathcal{E}, u_{i}\right)=\frac{1}{u_{i}} \sqrt{\frac{\mathcal{E}-V_{j}}{\mathcal{E}-V_{i}}+u_{i}^{2}-1} .
$$

Here $V_{i}$ is the electron potential in the layer $i$ and $u_{i}=\cos \theta_{i}$, where $\theta_{i}$ is the angle of incidence measured with respect to the axis $z$. The transmission and reflection coefficients obey the relations

$$
\begin{aligned}
& R_{i j}^{0}\left(\mathcal{E}, u_{i}\right)=R_{j i}^{0}\left(\mathcal{E}, u_{j}\right), \\
& T_{i j}^{0}\left(\mathcal{E}, u_{i}\right)=T_{j i}^{0}\left(\mathcal{E}, u_{j}\right),
\end{aligned}
$$

where

$$
u_{j}=\sqrt{1+\frac{\mathcal{E}-V_{i}}{\mathcal{E}-V_{j}}\left(u_{i}^{2}-1\right)} .
$$

Following Ref. [7] we include electron scattering processes at the interfaces between the magnetic and nonmagnetic films by the factors

$$
\begin{aligned}
S_{i j ; i}^{\sigma, \sigma^{\prime}} & =\bar{S}^{\sigma, \sigma^{\prime}} \exp \left[-4 \eta_{i j}^{2}\left(k_{i} u_{i}\right)^{2}\right], \\
S_{i j ; j}^{\sigma, \sigma^{\prime}} & =\bar{S}^{\sigma, \sigma^{\prime}} \exp \left[-\eta_{i j}^{2}\left(k_{i} u_{i}-k_{j} u_{j}\right)^{2}\right],
\end{aligned}
$$

where $\eta_{i j}$ is the amplitude of the geometrical roughness of the corresponding interface, $k_{i}$ is the Fermi wave vector in the $i$-th layer and the factors $\bar{S}^{\sigma, \sigma^{\prime}}$ describe effectively those scattering processes, which are not due to the geometrical roughness. In the following we assume the same roughness amplitude $\eta_{i j}=\eta_{\text {int }}$ for both interfaces. The boundary conditions at the interface between the $(i-1)$-th and $i$-th layers can be written as

and

$$
\begin{aligned}
& g_{i \sigma}^{+}\left(z_{0}, u_{i}\right) \equiv T_{i-1 i}^{0} S_{i-1 i ; i}^{\sigma, \sigma} g_{i-1 \sigma}^{+}\left(z_{0}, u_{i-1}\right)+T_{i-1 i}^{0} S_{i-1 i ; i}^{\sigma,-\sigma} g_{i-1-\sigma}^{+}\left(z_{0}, u_{i-1}\right) \\
& \quad+R_{i i-1}^{0} S_{i i-1 ; i}^{\sigma, \sigma} g_{i \sigma}^{-}\left(z_{0}, u_{i}\right)+R_{i i-1}^{0} S_{i i-1 ; i}^{\sigma,-\sigma} g_{i-\sigma}^{-}\left(z_{0}, u_{i}\right)
\end{aligned}
$$




$$
\begin{aligned}
& g_{i-1 \sigma}^{-}\left(z_{0}, u_{i-1}\right)=T_{i i-1}^{0} S_{i i-1 ; i-1}^{\sigma, \sigma} g_{i \sigma}^{-}\left(z_{0}, u_{i}\right)+T_{i i-1}^{0} S_{i i-1 ; i-1}^{\sigma,-\sigma} g_{i-\sigma}^{-}\left(z_{0}, u_{i}\right) \\
& +R_{i-1 i}^{0} S_{i-1 i ; i-1}^{\sigma, \sigma} g_{i-1 \sigma}^{+}\left(z_{0}, u_{i-1}\right)+R_{i-1 i}^{0} S_{i-1 i ; i-1}^{\sigma, \sigma} g_{i-1-\sigma}^{+}\left(z_{0}, u_{i-1}\right),
\end{aligned}
$$

where $z_{0}$ is the position of the interface. The terms with the factors $\bar{S}^{\sigma,-\sigma}$ describe effectively those spin-flip scattering processes, which conserve the in-plane electron momentum. In the following we introduce the notation: $\bar{S}^{\sigma,-\sigma}=\bar{S}^{-\sigma, \sigma} \equiv \bar{S}_{\text {int }}^{+-}$and $\bar{S}^{\sigma, \sigma} \equiv \bar{S}_{\text {int }}^{\sigma}$.

Similar boundary conditions also hold for the free surfaces. One can obtain them from Eqs. (10) and (11) by extending the layer index $i$ to include the outer half-spaces ( $i=0$ and $i=4)$, and by taking into account the fact that the distribution functions vanish exactly for $i=0$ and $i=4$ and the appropriate reflection coefficients are equal to unity. The surfaces are assumed to be statistically similar with the same roughness amplitude $\eta_{\text {sur. }}$. The corresponding factors $\bar{S}^{\sigma, \sigma^{\prime}}$ will be denoted as $\bar{S}^{\sigma, \sigma}=\bar{S}_{\text {sur }}^{\sigma} \equiv p_{\sigma}$ and $\bar{S}^{\sigma,-\sigma}=\bar{S}^{-\sigma, \sigma}=\bar{S}_{\text {sur }}^{+-} \equiv p_{+- \text {. }}$.

We assume the bottom of the electron band in the nonmagnetic film $\left(V_{2}=0\right)$ as the zero of energy. In the following numerical calculations we assumed the band edge in the magnetic films at $1.56 \mathrm{eV}$ and the Fermi energy at $8.54 \mathrm{eV}$. We also assumed $m=4 m_{0}$, where $m_{0}$ is the free electron mass, and introduced the electron mean free paths $\lambda_{\mathrm{m} \sigma}, \lambda_{\mathrm{m}+-}, \lambda_{\mathrm{n}}$ and $\lambda_{\mathrm{n}+-}$, which are determined by the corresponding Fermi velocities and relaxation times $\left(\lambda=v_{\mathrm{F}} \tau\right)$. For convenience, we also introduced the average mean free path in the magnetic films, $\bar{\lambda}_{\mathrm{m}}=$ $\left(\lambda_{\mathrm{m}+}+\lambda_{\mathrm{m}-}\right) / 2$.

Figure 1 presents the influence of bulk spin-mixing scattering processes. The relative resistance change, $\Delta R / R_{\uparrow \uparrow}=\left(R_{\uparrow \downarrow}-R_{\uparrow \uparrow}\right) / R_{\uparrow \uparrow}\left(R_{\uparrow \uparrow}\right.$ and $R_{\uparrow \downarrow}$ are the

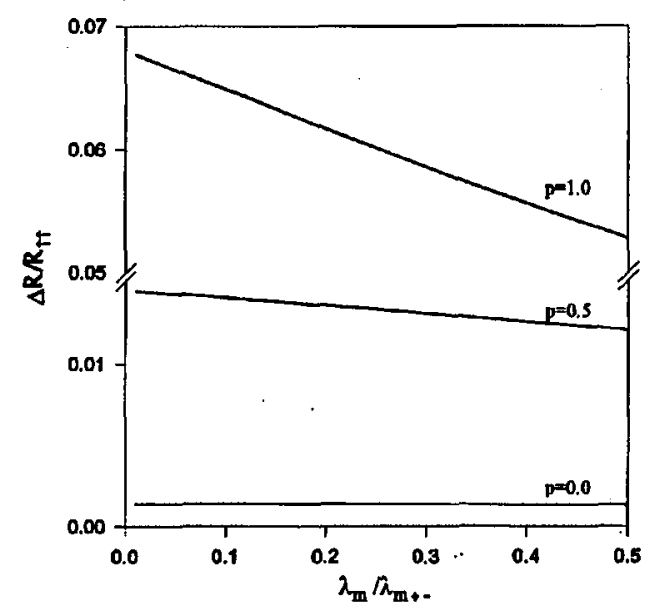

Fig. 1. $\Delta R / R_{\uparrow \uparrow}$ vs. $\bar{\lambda}_{\mathrm{m}} / \lambda_{\mathrm{m}+-}$ for $p$ as indicated. The other parameters are: $d_{\mathrm{m}}=$ $d_{\mathrm{n}}=20 \AA, \bar{S}_{\mathrm{int}}^{ \pm}=0.9, \bar{S}_{\mathrm{int}}^{+-}=0, p_{+-}=0, \eta_{\mathrm{int}}=1 \AA, \eta_{\mathrm{sur}}=0, \lambda_{\mathrm{n}}=\bar{\lambda}_{\mathrm{m}}=500 \AA$, $\lambda_{\mathrm{n}+-} / \lambda_{\mathrm{m}+-}=2$ and $\lambda_{\mathrm{m}-} / \lambda_{\mathrm{m}+}=8$. 


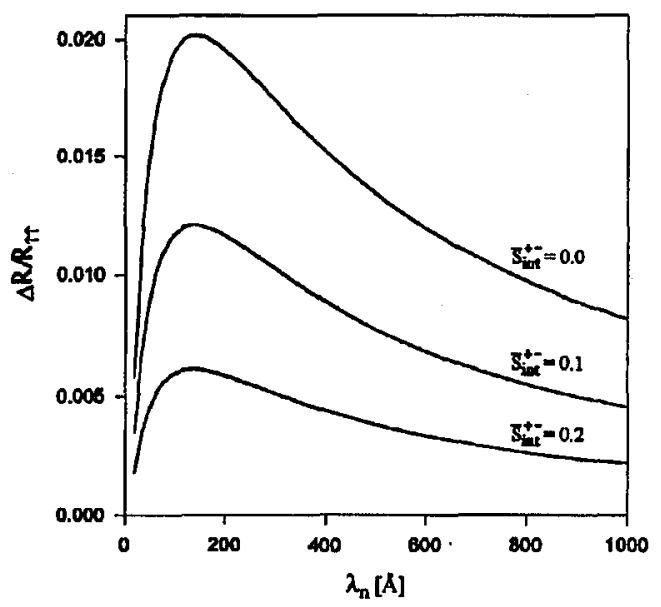

Fig. 2. $\Delta R / R_{\uparrow \uparrow}$ vs. mean free path $\lambda_{\mathrm{n}}$ in the nonmagnetic spacer. The other parameters are: $d_{\mathrm{m}}=d_{\mathrm{n}}=20 \AA, \bar{S}_{\text {int }}^{ \pm}=0.9-\bar{S}_{\text {int }}^{+-}=, p_{ \pm}=0.9, p_{+-}=0, \eta_{\text {int }}=\eta_{\text {sur }}=1.0 \AA$, $\lambda_{m-} / \lambda_{m+}=5, \lambda_{-}=\bar{\lambda}_{m}$ and $\lambda_{m+-} / \bar{\lambda}_{m}=\lambda_{n+-} / \lambda_{n}=50$.

resistances respectively in the parallel and antiparallel configurations of the film magnetizations) is shown there versus $\bar{\lambda}_{\mathrm{m}} / \lambda_{\mathrm{m}+-}$ for $p_{+-}=0$ and $p_{ \pm}=p$, with $p$ as indicated. The case $p=0$ corresponds to a sandwich structure with rather rough surfaces, when each electron incident on a surface is diffusely scattered. The case $p=1$, on the other hand, corresponds to the case where all electrons incident on the surfaces are specularly reflected. This is equivalent to an infinite superlattice with doubled thickness of the magnetic films. The spin-valve magnetoresistance decreases approximately linearly with increasing $1 / \lambda_{\mathrm{m}+-}$ (for a constant $\bar{\lambda}_{\mathrm{m}}$ ).

In Fig. 2 the relative resistance change is shown versus the electron mean free path in the nonmagnetic layer. Only the interface spin-mixing processes are assumed there, with $\bar{S}_{\text {int }}^{+-}$as indicated. It is clearly evident that SVMR is reduced by the interfacial electron-momentum-conserving spin-flip scattering processes.

The authors acknowledge support through the research project No. 2 P 30209107.

\section{References}

[1] J. Barnaś, A. Fuss, R.E. Camley, P. Grunberg, W. Zinn, Phys. Rev. B 42, 8110 (1990).

[2] B. Dieny, J. Phys., Condens. Matter 4, 8009 (1992).

[3] P.M. Levy, S. Zhang, A. Fert, Phys. Rev. Lett. 65, 1643 (1990).

[4] A. Fert, J. Phys., Solid State Phys. 2, 1784 (1969).

[5] J.L. Duvail, A. Fert, L.G. Pereira, D.K. Lottis, J. Appl. Phys. 75, 7070 (1994).

[6] J. Barnaś, O. Baksalary, J. Phys., Condens. Matter 7, 6437 (1995).

[7] R.Q. Hood, L.M. Falicov, D.R. Penn, Phys. Rev. B 49, 368 (1994). 